\title{
ESFORÇOS EM FERRAMENTAS ROMPEDORAS DE SOLO DE SEMEADORAS DE PLANTIO DIRETO
}

\author{
Loads in furrow opening tools for seeders on no-tillage systems
}

Renildo Luiz Mion', Sérgio Hugo Benez²

\begin{abstract}
RESUMO
A avaliação individual de mecanismos de abertura de sulco de semeadoras de plantio direto auxilia as empresas no dimensionamento de ferramentas rompedoras do solo, que buscam menor requerimento energético e a diminuição da área de solo mobilizado. Portanto, objetivou-se, neste trabalho, avaliar os esforços de cinco diferentes mecanismos de abertura de sulco de semeadoras de plantio direto, variando a carga vertical. O experimento foi instalado num solo classificado como Nitossolo Vermelho Distroférrico. O delineamento experimental utilizado foi o de blocos ao acaso, em esquema fatorial $5 \times 4$, constituídos dos seguintes fatores: cinco mecanismos de abertura de sulco (disco liso - DL, disco corrugado - DC, disco ondulado - DO, disco duplo - DD e haste sulcadora - F) e quatro cargas verticais $(750,1500,2250$ e $3000 \mathrm{~N})$. Para mensurar as forças nos tratamentos estudados foi utilizado um dinamômetro com sensibilidade nas direções horizontal, lateral, vertical. A avaliação mostrou que a interação entre mecanismos de abertura de sulco e carga vertical não foi significativa, entretanto os fatores foram significativos quando analisados individualmente. A haste promoveu a maior mobilização do solo com menor requerimento de esforço horizontal, atingindo as maiores profundidades de trabalho. O trabalho mostrou que a carga vertical influenciou a profundidade de trabalho dos mecanismos de abertura de sulco. Os mecanismos de abertura de sulco do tipo disco necessitam de maior força vertical para penetrar no solo.
\end{abstract}

Termos para indexação: Mecanismo de abertura de sulco, profundidade de trabalho, esforço tridimensional, dinamômetro.

\begin{abstract}
The individual evaluation of mechanisms for opening furrows to drop the seeds in a no-tillage seeders cultivation assists the companies in the sizing of opening tools of the ground, that search for minor energy petition and for diminishing the ground area mobilized. Therefore this work had the aim of evaluate the efforts of five different mechanisms of opening of ridge of seeding of direct plantation varyng the vertical load. The experiment was installed in one ground classified as Dystroferric Red Nitosol. The used experimental delineation was of the one randomized blocks in a factorial project $5 \times 4$, constituted a the following factors: five mechanisms of furrow opening (even disk - DL, corrugated disk - DC, ondulated disk - DO, double disk - DD and furrow rod - F) and four vertical loads $(750,1500,2250$ and $3000 \mathrm{~N})$. To measure the forces in the studied treatments a dynamometer with sensitivity in the directions was used horizontal, lateral and vertical direction. The evaluation showed that the interaction between mechanisms of opening of ridge and vertical load was not significant, however the factors were significant when analyzed individually. The connecting rod promoted mobilization of the ground with smaller draft of horizontal effort reaching depths of work. The work showed that the vertical load influenced the depth of work of the mechanisms of ridge opening. The mechanisms of ridge opening of record type need vertical force to penetrate into the ground.
\end{abstract}

Index terms: Mechanism of opening of ridge, depth of work, 3-D load, dynamometer.

(Recebido em 30 de março de 2007 e aprovado em 15 de maio de 2008)

\section{INTRODUÇÃO}

Nas últimas décadas a agricultura tem experimentado profundas transformações relacionadas aos aspectos técnicos, econômicos, políticos e sociais. As estratégias de manejo de solos estão sendo submetidas a significativas remodelações face às novas informações, tendências e conhecimentos recentemente gerados dentro do enfoque sistêmico de produção, destacando-se o sistema plantio direto que passa por uma grande expansão em várias regiões do Brasil.
O cultivo de culturas em áreas sob o sistema de plantio direto no mundo é da ordem de 94,6 milhões de hectares. Desse total, o Brasil participa com 24,95\%, representando, 23,6 milhões de ha; os Estados Unidos com $26,74 \%$, ou seja, 25,3 milhões de ha, somando mais de $50 \%$ da área cultivada. (ROBERTS \& JOHNSTON, 2006).

Os mecanismos de abertura de sulco são elementos mecânicos utilizados em semeadoras, a maioria dos quais empregados para assegurar a deposição de sementes e adubos no solo em ótimas condições para uma boa germinação e desenvolvimento. Considerando que as

\footnotetext{
Engenheiro Agrícola, Doutor - Departamento de Engenharia Agrícola/DENA - Universidade Federal do Ceará/UFC - Campus do Pici, Bloco 804 60455-760 - Fortaleza, CE - rmion@ufc.br

${ }^{2}$ Engenheiro Agrônomo, Doutor, Professor Titular - Departamento de Engenharia Rural/DER - Faculdade de Ciências Agronômicas/FCA - Universidade Estadual Paulista/UNESP - Fazenda Experimental Lageado - Cx. P. 237 - 18603-970 - Botucatu, SP - benez@fca.unesp.br
} 
condições para o desenvolvimento das culturas dependem do tipo de solo, teor de água, etc., é óbvio que o projeto de mecanismos não pode ser limitado a um único tipo capaz de ser usado para qualquer situação, dessa forma existem vários mecanismos de abertura de sulco empregados em semeadoras de plantio direto.

Para Germino \& Benez (2006), a profundidade de trabalho das hastes sulcadoras tem importante influência na força lateral. Os mesmos autores salientam que, nas profundidades de 230 e $280 \mathrm{~mm}$, obtiveram os menores esforços laterais quando comparados às profundidades de 120 e $330 \mathrm{~mm}$.

De acordo com Portella \& Faganello (1984) os mecanismos de abertura de sulco de semeadoras dotadas com sistemas de discos exigiram menor força para tração e maior força para penetração que as equipadas com sistemas de haste. Para Chang et al. (1983), a profundidade de penetração da haste sulcadora de uma semeadoraadubadora depende do seu ângulo de inclinação associada a uma força vertical atuando de baixo para cima.

Os sulcadores de disco, conforme Colombino et al. (1985), possuem penetração negativa, ou seja, para penetrar no solo necessitam uma carga superior à componente vertical de reação do mesmo.

Para MacIntire et al. (1986), sulcadores do tipo discos possuem uma capacidade razoável de corte dos resíduos e de penetração em solos argilosos, quando possuem pequeno diâmetro. Entretanto, seu desempenho não é totalmente satisfatório, devido à variação da profundidade de trabalho ocorrendo uma tendência inerente dos discos flutuarem sobre camadas duras do solo. Em solos de textura arenosa, a profundidade de semeadura desejada nem sempre é obtida, devido ao desmoronamento prematuro das laterais do sulco; em solos argilosos, os lados dos sulcos podem ser compactados, resultando em baixos índices de germinação.

Smith et al. (1967) relatam o comportamento dos dispositivos de abertura de sulcos do tipo discos com formato ondulado: à medida que a profundidade de trabalho aumenta, a necessidade de energia para tração por unidade de volume também aumenta. Esse aumento é atribuído ao aumento na área de contato entre o disco de corte e o solo, ocasionando maior requerimento de energia. Os mesmos autores afirmam que discos de corte planos, com diferentes diâmetros são os mais comuns em projetos de semeadoras, salientando que as semeadoras devem permitir a adição de peso extra para obter penetração em solos não mobilizados.

Segundo Faganello et al. (1992) as semeadoras de plantio direto podem apresentar discos de corte de diferentes diâmetros e formatos (plano, liso, ondulado, estriado, corrugado). Os mesmos têm a função de cortar os restos culturais e abrir um sulco, facilitando a ação dos mecanismos depositores de fertilizantes e/ou sementes. Contudo, os mecanismos normalmente adotados pelas semeadoras nacionais de plantio direto são ineficientes para operar nas diferentes condições de solos e restos culturais, necessitando do desenvolvimento de mecanismos de abertura de sulco mais eficientes. O mesmo autor salienta que os discos planos e estriados cortam melhor os restos culturais depositados na superfície do solo e demandam menos peso para penetração, contrastando com os discos estriados e ondulados que possuem maior superfície específica, porém os discos de corte estriados e ondulados estão menos sujeitos à patinagem.

De acordo com Janke \& Erbach (1985), no sistema plantio direto, os dispositivos de abertura de sulco e de controle de profundidade quando são submetidos a cargas, sofrem um esforço maior no rompimento do solo devido à variação da resistência do solo à penetração, irregularidades da superfície e à presença da cobertura vegetal.

Objetivou-se, neste trabalho, avaliar forças em diferentes direções variando os mecanismos de abertura de sulco de semeadoras de plantio direto em função da carga vertical.

\section{MATERIALE MÉTODOS}

O experimento foi conduzido em área da Fazenda Experimental Lageado, pertencente à Faculdade de Ciências Agronômicas FCA/UNESP, Campus de Botucatu - SP, cuja localização geográfica está definida pelas coordenadas $22^{\circ}$ 41' latitude Sul e 48 $34^{\prime}$ ' de longitude Oeste, com altitude média de $770 \mathrm{~m}$. A área estava sendo manejada no sistema plantio direto, com vegetação predominante de restos culturais de soja. O solo da área foi classificado como Nitossolo Vermelho Distroférrico com relevo plano a ondulado e declividade média de $4 \%$.

Os resultados da análise granulométrica na camada de 0 - $150 \mathrm{~mm}$ apresentaram os valores médios de 244, 206 e $550 \mathrm{~g} \mathrm{~kg}^{-1}$ para areia, silte e argila, respectivamente, sendo classificado como de textura argilosa. A densidade do solo apresentou valores médios de $1,25 \mathrm{~g} \mathrm{~kg}^{1}{ }^{1} \mathrm{e}$ o teor de água do solo no momento do experimento foi de $25,52 \%$.

Foi desenvolvida uma barra porta-ferramenta, acoplada ao sistema hidráulico do trator com dinamômetro para acoplamento das ferramentas ensaiadas.

Os mecanismos de abertura de sulco utilizados para a realização deste ensaio, foram: disco de corte liso com $381 \mathrm{~mm}$ de diâmetro, $3 \mathrm{~mm}$ de espessura e ângulo de fio de 
$10,5^{\circ}$, disco duplo com dois discos planos de $381 \mathrm{~mm}$ de diâmetro, $3 \mathrm{~mm}$ de espessura, posicionados um em relação ao outro a um ângulo de $10,5^{\circ}$, com eixo desencontrado; disco corrugado de $355 \mathrm{~mm}$ de diâmetro, $3 \mathrm{~mm}$ de espessura e ângulo de fio de $5^{\circ}$; disco ondulado com $402 \mathrm{~mm}$ de diâmetro, $3 \mathrm{~mm}$ de espessura e ângulo de fio de $12^{\circ}$; haste sulcadora com as seguintes dimensões: espessura de 9 $\mathrm{mm}$, largura da ponteira de $28 \mathrm{~mm}$, ângulo vertical da ponteira $18,5^{\circ}$ e ângulo horizontal de $16^{\circ}$.

Os sinais gerados pelos sensores foram armazenados e monitorados por um sistema de aquisição de dados fabricado pela Campbell Scientific, Inc. modelo "CR 21 X micrologger". A velocidade de deslocamento foi determinada por meio de uma unidade de radar fabricada pela Dickey-John, modelo DjRVS II, instalado lateralmente ao trator.

Para determinar a profundidade de trabalho dos mecanismos de abertura de sulco utilizou-se um sistema composto por uma cremalheira acoplada a um potenciômetro de 5 volts e 10 voltas (para medir o deslocamento vertical da haste).

O experimento foi constituído por 20 tratamentos em blocos ao acaso com quatro repetições, totalizando 80 parcelas experimentais. A parcela possuía $15 \mathrm{~m}$ de comprimento e $3 \mathrm{~m}$ de largura, perfazendo um total de 45 $\mathrm{m}^{2}$. Entre os blocos foi deixado um carreador com $10 \mathrm{~m}$ para manobras e estabilização do equipamento, antes do inicio da aquisição de dados.

Os tratamentos foram compostos pelos seguintes mecanismos de abertura de sulco DD = disco duplo, $\mathrm{DL}=$ disco liso, $\mathrm{DC}=$ disco corrugado, $\mathrm{DO}=$ disco ondulado e $\mathrm{F}=$ haste sulcadora e, as carga verticais foram de $750 \mathrm{~N}$, $1500 \mathrm{~N}, 2250 \mathrm{~N}$ e $3000 \mathrm{~N}$.

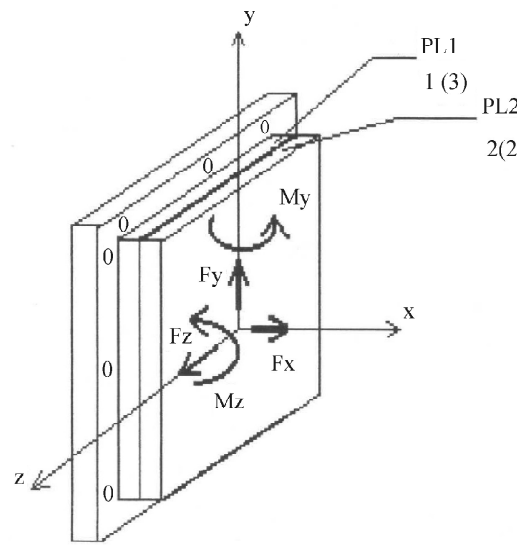

PL1: Fy; Fx; Mz

I'L2: Hz; My
O sistema de aquisição de dados "micrologger $21 \mathrm{X}$ " foi usado nos experimentos para monitorar e gravar continuamente, com uma freqüência de $10 \mathrm{~Hz}$ todos os sinais gerados pelos sensores.

A velocidade média de deslocamento no experimento foi de $5,19 \mathrm{~km} \mathrm{~h}^{-1}$, que foi obtida pelo método indireto através da frequiência de aquisição $(10 \mathrm{~Hz})$ do coletor de dados, sendo de dez registros por segundo. $\mathrm{O}$ tempo gasto para percorrer cada parcela correspondeu ao número de registros obtidos, que foram armazenados no intervalo, sendo a velocidade média de deslocamento calculada pela Equação 1:

$$
\text { Vel }=\left(\frac{L}{t}\right) \times 3,6
$$

Em que:

Vel $=$ velocidade de deslocamento $\left(\mathrm{km} \mathrm{h}^{-1}\right)$

$\mathrm{L}=$ comprimento da parcela $(15 \mathrm{~m})$

$\mathrm{t}=$ tempo gasto para percorrer a parcela (s)

$3,6=$ constante

Para mensurar as forças dos mecanismos de abertura de sulco foi utilizado um dinamômetro marca MSI, com capacidade de $25000 \mathrm{~N}$, instrumentado com extensômetros elétricos de resistência ligados em pontes de Wheatstone, um para cada força medida. A capacidade do dinamômetro é de $25000 \mathrm{~N}$ para cada variável, conforme Figura 1. As principais características do dinamômetro são: freqüência de ressonância superior a $2,0 \mathrm{kHz}$; não linearidade de $0,25 \%$ do fundo de escala; repetibilidade de $0,15 \%$ do fundo de escala e "crosstalk" menor que $2 \%$ para um canal com carga e outro sem carga.

PL 1 = placa para medir as forças vertical (Fy), horizontal (Fx) e momento lateral $(\mathrm{Mz})$.

PL 2 = placa para medir as forças lateral $(\mathrm{Fz})$ e momento lateral (My).

Figura 1-Dinamômetro 
A determinação das variáveis foi calculada por meio da média aritmética de todos os valores armazenados em cada parcela pelo número de dados coletados, de acordo com a freqüência utilizada nos sensores, da seguinte forma:

$$
F=\sum_{i=1}^{n} \frac{F i}{n} \times 10
$$

$$
\begin{aligned}
& \text { em que: } \\
& \mathrm{F}=\text { força média }(\mathrm{N}) \\
& \mathrm{Fi}=\text { força instantânea }(\mathrm{kgf}) \\
& \mathrm{n}=\text { número de dados registrados } \\
& 10=\text { constante }
\end{aligned}
$$

\section{RESULTADOS E DISCUSSÃO}

Pela Tabela 1, observa-se que o mecanismo de abertura de sulco do tipo haste, obteve menor requerimento de força independente da carga vertical aplicada. Isso mostra claramente o modo de ação do mecanismo sulcador do tipo haste, em que apenas uma parte (ponta) toca no solo e é a responsável pela resistência oferecida ao corte, sendo que o solo flui pelas laterais da haste sem alterar a força horizontal requerida, concordando com Portella (1983).

Os mecanismos de abertura de sulco do tipo disco em relação à haste, necessitaram de maior força horizontal devido a sua função de penetrar, cortar o solo e a palha da superfície, vencer a resistência ao rolamento e ao atrito nas laterais dos disco.

Conforme os dados apresentados na Tabela 2, observa-se que a carga vertical de $750 \mathrm{~N}$, o mecanismo de abertura de sulco do tipo disco liso obteve a maior força vertical em relação aos demais mecanismos de abertura de sulco, esse pode ser explicado pela presença dos restos vegetais na superfície do solo e a função do disco em penetrar no solo. Esses valores estão próximos aos encontrados por Morrison Júnior et al. (1996), realizando pesquisa em solos argilosos. Observa-se que o mecanismo de abertura de sulco do tipo haste teve um desempenho diferente dos demais, devido à ocorrência de sucção e profundidade de trabalho, concordando com Portella \& Faganello (1984) em que as hastes necessitam de menos força para penetração do que os discos e Chang et al. (1983) concluíram que as hastes sulcadoras dependem do

Tabela 1 - Valores médios de força horizontal (N) em função da carga vertical.

\begin{tabular}{cccccc}
\hline Tratamento & \multicolumn{5}{c}{ CARGA VERTICAL $(\mathrm{N})$} \\
\cline { 2 - 5 } & 750 & 1500 & 2250 & 3000 & Média \\
\hline DO & $1369,90 \mathrm{aC}$ & $1780,90 \mathrm{aB}$ & $1935,00 \mathrm{aAB}$ & $2172,10 \mathrm{aA}$ & 1809,00 \\
DC & $1139,30 \mathrm{aA}$ & $1241,70 \mathrm{bA}$ & $1431,40 \mathrm{bA}$ & $1403,10 \mathrm{cA}$ & 1515,80 \\
DL & $1286,00 \mathrm{aA}$ & $1520,30 \mathrm{abA}$ & $1544,70 \mathrm{bA}$ & $1403,20 \mathrm{cA}$ & 1304,70 \\
F & $664,10 \mathrm{bB}$ & $675,20 \mathrm{cB}$ & $883,40 \mathrm{cB}$ & $1208,70 \mathrm{cA}$ & 857,80 \\
DD & $1348,10 \mathrm{aB}$ & $1589,70 \mathrm{aB}$ & $1428,80 \mathrm{bB}$ & $1828,30 \mathrm{bA}$ & 1476,80 \\
\hline Média & 1161,70 & 1361,70 & 1444,80 & 1608,00 & 1393,80 \\
\hline
\end{tabular}

Médias seguidas de mesmas letras minúsculas nas colunas e maiúsculas na linha não diferem entre si, pelo Teste de Tukey, ao nível de 5\% de probabilidade.

Tabela 2 - Valores médios de força vertical (N) em função da carga vertical.

\begin{tabular}{cccccc}
\hline Tratamento & \multicolumn{5}{c}{ CARGA VERTICAL (N) } \\
\cline { 2 - 5 } & 750 & 1500 & 2250 & 3000 & Média \\
\hline DO & $-893,60 \mathrm{abB}$ & $-1252,00 \mathrm{aA}$ & $-1348,30 \mathrm{aA}$ & $-1234,60 \mathrm{abA}$ & $-1182,10$ \\
DC & $-843,30 \mathrm{bA}$ & $-1104,60 \mathrm{aA}$ & $-1008,80 \mathrm{bA}$ & $-1034,10 \mathrm{bA}$ & $-1170,80$ \\
DL & $-1170,90 \mathrm{aA}$ & $-1354,10 \mathrm{aA}$ & $-1290,20 \mathrm{bcA}$ & $-1163,00 \mathrm{abA}$ & $-997,70$ \\
F & $14,60 \mathrm{cA}$ & $-38,10 \mathrm{bA}$ & $-14,80 \mathrm{cA}$ & $-3,30 \mathrm{cA}$ & $-10,40$ \\
DD & $-999,80 \mathrm{abB}$ & $-1243,60 \mathrm{aAB}$ & $-1045,40 \mathrm{bcB}$ & $-1394,30 \mathrm{aA}$ & $-1244,50$ \\
\hline Média & $-778,60$ & $-998,50$ & $-941,50$ & $-965,80$ & $-921,10$ \\
\hline
\end{tabular}

Médias seguidas de mesmas letras minúsculas nas colunas e maiúsculas na linha não diferem entre si, pelo Teste de Tukey, ao nível de $5 \%$ de probabilidade. 
ângulo de inclinação associado a uma força vertical, atuando de baixo para cima.

Considerando os dados apresentados na Tabela 3, observa-se que o disco ondulado apresentou valores de força lateral superior aos demais sulcadores para as cargas verticais de 1500,2250 e $3000 \mathrm{~N}$, mostrando que esse comportamento, provavelmente, deve-se ao desenho da ferramenta. Isso nos mostra tendência do equipamento possuir uma força lateral, podendo ser influenciada tanto pela declividade quanto pela direção do trator, o que pode causar problemas para as semeadoras quando estiverem trabalhando em local com declividade. Isso também pode ter ocorrido pela interação solo-metal causando fricção e gerando forças laterais aumentando dessa forma a resistência ao rolamento e desgaste dos discos.

Para o mecanismo sulcador do tipo haste sulcadora, a força lateral ocorreu para o lado oposto aos discos, porém com menor intensidade. Esses valores estão próximo aos encontrados por Germino \& Benez (2006), trabalhando com cargas verticais de $3000 \mathrm{~N}$ e diferentes profundidades.

$\mathrm{Na}$ Tabela 4, são apresentados os valores médios da variação da profundidade dos diferentes mecanismos de abertura de sulco. Observa-se que independente da carga sobre a barra porta-ferramenta, o mecanismo de abertura de sulco do tipo haste atingiu maiores valores de profundidade; esse desempenho está de acordo com o observado por Araújo et al. (1999), em que a haste tende a puxar o equipamento para baixo. Observa-se que a carga vertical $3000 \mathrm{~N}$ proporcionou as maiores profundidades, mostrando que no momento da semeadura deve-se procurar manter o reservatório de sementes e adubos cheios, para evitar a variação da profundidade de semeadura com diferentes cargas, conforme salientam ABNT (1994) e Mialhe (1996), os níveis do reservatório para ensaio de semeadoras devem operar constantemente com $50 \%$ da sua capacidade volumétrica.

Os valores da área de solo mobilizado, apresentados na Tabela 5, foram diferentes para os mecanismos de abertura de sulco estudados, observando que o tipo haste proporcionou maior área mobilizada em relação aos demais, o que pode ser explicado pela maior profundidade de trabalho e largura da ponteira desse mecanismo sulcador, salientando que a carga vertical de $3000 \mathrm{~N}$ mobilizou $30 \%$ a mais de solo em relação à carga vertical de $750 \mathrm{~N}$, concordando com Silva (2007), que observou que, quanto maior a carga vertical, maior é a área de solo mobilizada.

Tabela 3 - Valores médios de força lateral (N) em função da carga vertical.

\begin{tabular}{cccccc}
\hline \multirow{2}{*}{ Tratamento } & \multicolumn{5}{c}{ CARGA VERTICAL (N) } \\
\cline { 2 - 5 } & 750 & 1500 & 2250 & 3000 & Média \\
\hline DO & $-1924,30 \mathrm{aC}$ & $-2381,70 \mathrm{aB}$ & $-2375,30 \mathrm{aB}$ & $-2678,20 \mathrm{aA}$ & $-2339,90$ \\
DC & $-1252,60 \mathrm{bB}$ & $-1433,10 \mathrm{cAB}$ & $-1592,30 \mathrm{cA}$ & $-1539,70 \mathrm{cA}$ & $-1993,30$ \\
DL & $-1322,40 \mathrm{bB}$ & $-1686,50 \mathrm{cA}$ & $-1454,50 \mathrm{cAB}$ & $-1249,90 \mathrm{~dB}$ & $-1454,40$ \\
F & $178,10 \mathrm{cA}$ & $160,80 \mathrm{dA}$ & $214,50 \mathrm{dA}$ & $179,70 \mathrm{eA}$ & 183,20 \\
DD & $-1778,40 \mathrm{aB}$ & $-2008,30 \mathrm{bB}$ & $-1888,30 \mathrm{bB}$ & $-2298,20 \mathrm{bA}$ & $-1428,60$ \\
\hline Média & $-1220,10$ & $-1469,80$ & $-1419,20$ & $-1517,10$ & 1406,50
\end{tabular}

Médias seguidas de mesmas letras minúsculas nas colunas e maiúsculas na linha não diferem entre si, pelo Teste de Tukey, ao nível de $5 \%$ de probabilidade.

Tabela 4 - Valores médios de profundidade ( $\mathrm{mm})$ em função da carga vertical.

\begin{tabular}{cccccc}
\hline Tratamento & \multicolumn{5}{c}{ CARGA VERTICAL $(\mathrm{N})$} \\
\cline { 2 - 6 } & 750 & 1500 & 2250 & 3000 & Média \\
\hline DO & $49,00 \mathrm{bC}$ & $52,40 \mathrm{bB}$ & $57,00 \mathrm{bAB}$ & $61,00 \mathrm{bA}$ & 45,90 \\
DC & $38,00 \mathrm{cD}$ & $46,20 \mathrm{cC}$ & $54,50 \mathrm{bB}$ & $61,00 \mathrm{bA}$ & 38,80 \\
DL & $45,00 \mathrm{bC}$ & $51,70 \mathrm{bB}$ & $55,50 \mathrm{bB}$ & $66,00 \mathrm{bA}$ & 46,60 \\
F & $61,60 \mathrm{aB}$ & $62,90 \mathrm{aB}$ & $66,50 \mathrm{aB}$ & $72,00 \mathrm{aA}$ & 61,60 \\
DD & $44,60 \mathrm{bC}$ & $50,60 \mathrm{bB}$ & $54,50 \mathrm{bB}$ & $62,10 \mathrm{bA}$ & 45,00 \\
\hline Média & 47,20 & 52,70 & 57,70 & 64,40 & 55,50 \\
\hline
\end{tabular}

Médias seguidas de mesmas letras minúsculas nas colunas e maiúsculas na linha não diferem entre si, pelo Teste de Tukey, ao nível de $5 \%$ de probabilidade. 
Tabela 5 - Valores médios de área de solo mobilizada $\left(\mathrm{mm}^{2}\right)$ em função da carga vertical.

\begin{tabular}{cccccc}
\hline \multirow{2}{*}{ Tratamento } & \multicolumn{5}{c}{ CARGA VERTICAL $(\mathrm{N})$} \\
\cline { 2 - 5 } & 750 & 1500 & 2250 & 3000 & Média \\
\hline DO & $519,80 \mathrm{bD}$ & $611,50 \mathrm{bC}$ & $676,30 \mathrm{bA}$ & $658,40 \mathrm{bB}$ & 609,80 \\
DC & $409,80 \mathrm{~dB}$ & $304,60 \mathrm{eD}$ & $356,70 \mathrm{dC}$ & $489,20 \mathrm{eA}$ & 390,00 \\
DL & $383,40 \mathrm{eB}$ & $334,70 \mathrm{dD}$ & $366,00 \mathrm{dC}$ & $456,90 \mathrm{dA}$ & 385,20 \\
F & $587,20 \mathrm{aD}$ & $660,00 \mathrm{aC}$ & $700,50 \mathrm{aB}$ & $796,00 \mathrm{aA}$ & 685,90 \\
DD & $450,00 \mathrm{cC}$ & $584,80 \mathrm{cA}$ & $589,00 \mathrm{cA}$ & $536,40 \mathrm{cB}$ & 546,70 \\
\hline Média & 478,00 & 491,00 & 537,70 & 587,30 & 523,50 \\
\hline
\end{tabular}

Médias seguidas de mesmas letras minúsculas nas colunas e maiúsculas na linha não diferem entre si, pelo Teste de Tukey, ao nível de $5 \%$ de probabilidade.

Com relação aos mecanismos de discos: o disco ondulado mobilizou maior área quando comparado aos demais devido à área de contato solo-ferramenta, necessitando de maior peso para cortar o material da superfície do solo, resultando em sulcos mais largos e com conseqüente aumento na área e volume de solo mobilizado, fato esse observado também por Siqueira \& Casão Júnior (2004).

\section{CONCLUSÕES}

A haste promoveu a maior mobilização do solo com menor requerimento de esforço horizontal, atingindo as maiores profundidades de trabalho.

O trabalho mostrou que a carga vertical influenciou a profundidade de trabalho dos mecanismos de abertura de sulco.

Os mecanismos de abertura de sulco, do tipo disco, necessitam de maior força vertical para penetrar no solo.

\section{REFERÊNCIAS BIBLIOGRÁFICAS}

ARAÚJO, A. G.; CASÃO JÚNIOR, R.; RALISCH, R.; SIQUEIRA, R. Mobilização de solo e emergência de plantas na semeadura direta de soja (Glycine max L.) e milho (Zea mays L.) em solos argilosos. Engenharia Agrícola, v. 19, p. 226-237, 1999.

ASSOCIAÇÃO BRASILEIRA DE NORMAS TÉCNICAS. Projeto de norma 04: 015.06-004: semeadora de precisão: ensaio de laboratório: método de ensaio. Rio de Janeiro, 1994. 7 p.

CHANG, C. S.; PORTELLA, J. A.; KUMAGAI, I. Exigência da força nos sistemas que utilizam tração para o plantio direto. In: CONGRESSO BRASILEIRO DE ENGENHARIA AGRÍCOLA, 13., 1983, Rio de Janeiro. Resumos... Rio de
Janeiro: Sociedade Brasileira de Engenharia Agrícola, 1983. p. 130

COlOMBINO, A.; POLlACINO, J. C.; SOSA, R. O. Máquinas para implantacion de cultivos. Buenos Aires: Universidade de Buenos Aieres, 1985. 25 p. Apostila.

FAGANELLO, A.; KOCHHANN, R. A.; PORTELLA, J. A. Desenvolvimento de mecanismos de corte para semeadoras de plantio direto. In: CONGRESSO BRASILEIRO DE ENGENHARIA AGRÍCOLA, 21., 1992, Santa Maria. Anais... Santa Maria: Sociedade Brasileira de Engenharia Agrícola, 1992. p. 1262-1271.

GERMINO, R.; BENEZ, S. H. Ensaio comparativo em dois modelos de hastes sulcadoras para semeadorasadubadoras de plantio direto. Revista Energia na Agricultura, Botucatu, v. 21, n. 3, p. 85-92, 2006.

JANKE, J.; ERBACH, D. C. Seed depth distribuition in notill and strip till. ASAE Pap, v. 85, n. 1013, p. 1-12, 1985.

MAcINTIRE, D.; GRAY, A. G.; SHARP, M. J. The development and fields trials of the "A" blade coulter for introducing seed into the soil. Journal Agricultural Engineering Research, v. 41, p. 43-51, 1986.

MIALHE, L. G. Máquinas agrícolas: ensaios \& certificação. Piracicaba: FEALQ, 1996. 723 p.

MORRISON JÚNIOR, J. E.; HENDRICK III, J. G.; SCHAFER, R. L. Soil forces on coulter and disc-opener combinations. Transaction of ASAE, Amsterdam, v. 39, n. 3, p. 369-376, 1996.

PORTELLA, J. A. Um estudo preliminar das forças atuantes em elementos rompedores de semeadeiras diretas comerciais. 1983. 69 f. Dissertação (Mestrado) Universidade Estadual de Campinas, Campinas, 1983. 
PORTELlA, A. J.; FAGANELlO, A. Máquinas para semeadura direta: desempenho dos elementos rompedores de solo. Dirigente Rural, v. 23, p. 13-26, 1984.

ROBERTS, T. L.; JOHNSTON, A. M. Intensidade de cultivo, rotações e tecnologia de adubação para a produção sustentável de trigo: uma experiência norte-americana. Revista Plantio Direto, v. 93, 2006.

SILVA, P. R. A. Semeadora adubadoras: mecanismos de corte de palha e cargas verticais aplicadas. 2007. 93 f. Tese
(Doutorado) - Universidade Estadual Paulista, Botucatu, 2007.

SIQUEIRA, R.; CASÃO JÚNIOR, R. Trabalhador no cultivo de grãos e oleaginosas: máquinas para manejo de coberturas e semeadura no sistema de plantio direto. Curitiba: SENAR, 2004. (Coleção SENAR).

SMITH, E. M.; VERMA, B. P.; TAYLOR, T. H. Energy required to operate disk furrower in Kentuchy Bluegrass sad. Transaction of ASAE, Amsterdam, v. 10, n. 4, p. 333-336, 1967. 